\title{
BMJ
}

\section{Potential of an age adjusted D-dimer cut-off value to improve the exclusion of pulmonary embolism in older patients: $a$ retrospective analysis of three large cohorts}

\author{
Renée A Douma, physician, ${ }^{1}$ Grégoire le Gal, physician, ${ }^{2}$ Maaike Söhne, physician, ${ }^{1}$ Marc Righini, physician, ${ }^{3}$ \\ Pieter W Kamphuisen, physician, ${ }^{1}$ Arnaud Perrier, professor, ${ }^{4}$ Marieke J H A Kruip, physician, ${ }^{5}$ Henri \\ Bounameaux, professor, ${ }^{3}$ Harry R Büller, professor, ${ }^{1}$ Pierre-Marie Roy, professor $^{6}$
}

\begin{abstract}
Department of Vascular Medicine, Academic Medical Centre, Meibergdreef 9, $1105 \mathrm{AZ}$, Amsterdam, Netherlands

${ }^{2}$ Department of Internal Medicine and Chest Diseases, CHU la

Cavale Blanche, Equipe d'accueil 3878 (GETBO), Brest University

Hospital, 29609 Brest, France

${ }^{3}$ Division of Angiology and Haemostasis, Department of Internal Medicine, Geneva Faculty of Medicine, University of Geneva, CH-1211 Geneva, Switzerland

${ }^{4}$ Division of Internal General Medicine, Geneva Faculty of Medicine, University of Geneva, CH-1211 Geneva, Switzerland

${ }^{5}$ Department of Haematology, Erasmus Medical Centre, 'sGravendijkwal 230, 3015

CE Rotterdam, Netherlands

${ }^{6}$ Department of Emergency Medicine, University of Angers, F49933 Angers Cedex 9, France

Correspondence to: $\mathrm{R}$ A Douma

R.A.Douma@amc.uva.nl
\end{abstract}

Cite this as: $B M J$ 2010;340:c1475 doi:10.1136/bmj.c1475

\section{ABSTRACT}

Objectives In older patients, the the D-dimer test for pulmonary embolism has reduced specificity and is therefore less useful. In this study a new, age dependent cut-off value for the test was devised and its usefulness with older patients assessed.

Design Retrospective multicentre cohort study. Setting General and teaching hospitals in Belgium, France, the Netherlands, and Switzerland.

Patients 5132 consecutive patients with clinically suspected pulmonary embolism.

Intervention Development of a new D-dimer cut-off point in patients aged $>50$ years in a derivation set (data from two multicentre cohort studies), based on receiver operating characteristics (ROC) curves. This cut-off value was then validated with two independent validation datasets.

Main outcome measures The proportion of patients in the validation cohorts with a negative D-dimer test, the proportion in whom pulmonary embolism could be excluded, and the false negative rates.

Results The new D-dimer cut-off value was defined as (patient's age×10) $\mu \mathrm{g} / \mathrm{l}$ in patients aged $>50$. In 1331 patients in the derivation set with an "unlikely" score from clinical probability assessment, pulmonary embolism could be excluded in $42 \%$ with the new cut-off value versus $36 \%$ with the old cut-off value $(2500 \mu \mathrm{g} / \mathrm{l})$. In the two validation sets, the increase in the proportion of patients with a D-dimer below the new cut-off value compared with the old value was $5 \%$ and $6 \%$. This absolute increase was largest among patients aged > 70 years, ranging from $13 \%$ to $16 \%$ in the three datasets. The failure rates (all ages) were $0.2 \%(95 \% \mathrm{Cl} 0 \%$ to $1.0 \%)$ in the derivation set and $0.6 \%(0.3 \%$ to $1.3 \%)$ and $0.3 \%$ $(0.1 \%$ to $1.1 \%)$ in the two validation sets.

Conclusions The age adjusted D-dimer cut-off point, combined with clinical probability, greatly increased the proportion of older patients in whom pulmonary embolism could be safely excluded.

\section{INTRODUCTION}

Measurement of D-dimer concentration in the blood is a valuable tool in the diagnostic investigation of patients with suspected pulmonary embolism. A Ddimer concentration below the conventional cut-off point of $500 \mu \mathrm{g} / \mathrm{l}$ combined with a "low/intermediate" or "unlikely" clinical probability can safely rule out the diagnosis in about $30 \%$ of patients with suspected pulmonary embolism. ${ }^{1-3}$ However, the D-dimer concentration increases with age and its specificity for embolism decreases, which makes the test less useful to exclude pulmonary embolism in older patients. ${ }^{4-9}$ Indeed, the test is able to rule out pulmonary embolism in $60 \%$ of patients aged $<40$ years, but in only $5 \%$ of patients aged $>80 .^{8}$

If the D-dimer test is combined with an "unlikely" clinical probability ${ }^{10}$ to rule out pulmonary embolism, $10 \%$ of patients $>75$ years old versus $32 \%$ of patients of all ages do not need further diagnostic testing; the number needed to test for one negative test result is 10.6 and 3.1 , respectively. ${ }^{9}$ Raising the cut-off value to various points between $600 \mu \mathrm{g} / \mathrm{l}$ and $1000 \mu \mathrm{g} / \mathrm{l}$ increased specificity, but this came at the cost of safety, with more false negative test results. ${ }^{11}$ In this analysis, however, no stratification was made for clinical probability.

By constructing receiver operating characteristics (ROC) curves, we derived a new, age dependent Ddimer cut-off value and analysed its safety and clinical utility, in combination with clinical probability assessment, for the exclusion of pulmonary embolism in two large prospective cohort studies of patients with suspected pulmonary embolism. We then validated the outcome in two other large management studies.

\section{METHODS}

Derivation set

We analysed the combined data from two prospective multicentre cohort studies, which included 1721 consecutive outpatients with suspected pulmonary embolism. ${ }^{12}$ These outcome studies were designed to evaluate diagnostic strategies for pulmonary embolism, combining clinical probability assessment, D-dimer measurement, lower limb venous compression ultrasonography, and helical computed tomography. 
Briefly, all consecutive patients admitted to the emergency department of four general and teaching hospitals were included if they had a clinical suspicion of pulmonary embolism. The inclusion and exclusion criteria and the results of the two studies have been published previously. ${ }^{12}$

The first study, conducted at Geneva University Hospital, Geneva, and Centre Hospitalier Universitaire Vaudois, Lausanne, both in Switzerland, and at Angers University Hospital, Angers, France, between October 2000 and June 2002, comprised 965 patients. ${ }^{12}$ The second study, conducted at Geneva University Hospital, Angers University Hospital, and Hôpital Européen Georges Pompidou, Paris, France, between September 2002 and October 2003, comprised 756 patients. ${ }^{1}$ Both studies were approved by the institutional review boards of each participating institution and written informed consent was obtained from all patients.

All patients underwent a sequential diagnostic investigation, including plasma D-dimer measurement by an enzyme linked immunosorbent assay (rapid ELISA assay, VIDAS D-Dimer Exclusion, Biomérieux, Marcy-l'Etoile, France). For each patient, the Geneva score ${ }^{13}$ was assessed to assign the patient to a clinical probability category - with possible override by implicit assessment in case the result conflicted with the assessor's clinical judgment. ${ }^{14}$ Variables included in the Wells clinical prediction rule for pulmonary embolism ${ }^{10}$ were also systematically and prospectively collected, allowing calculation of the Wells score.

Pulmonary embolism was ruled out by $(a)$ a D-dimer concentration $<500 \mu \mathrm{g} / \mathrm{l}$, except in patients with a high clinical probability, in the second study; $(b)$ negative results from lower limb venous compression ultrasonography and from helical computed tomography in patients with a low or intermediate clinical probability of pulmonary embolism; or (c) by a normal ventilationperfusion lung scan or a normal pulmonary angiogram in patients with a high clinical probability or with inconclusive helical computed tomogram. Pulmonary embolism was established by $(a)$ finding a proximal deep vein thrombosis on lower limb ultrasonography; (b) a positive result from helical computed tomography; or $(c)$ a high probability ventilation-perfusion lung scan or a positive pulmonary angiogram in high clinical probability patients with negative results from both compression ultrasonography and helical computed tomography, and in patients with inconclusive computed tomogram.

Patients were followed up by their family physicians and were interviewed by telephone by one of the study coordinators at the end of three months' follow-up. The outcome was an estimate of the three month thromboembolic risk in patients in whom pulmonary embolism was considered ruled out by the initial diagnostic investigations and who did not receive anticoagulants during follow-up. Confirmation of venous thromboembolic events during follow-up were established with the usual criteria. ${ }^{1212}$
Validation set 1

For the first validation set, data from a third prospective multicentre cohort study were used. ${ }^{2}$ This study evaluated the clinical effectiveness of a simplified algorithm using the dichotomised Wells rule, D-dimer testing, and computed tomography in patients with suspected pulmonary embolism. Briefly, all consecutive inpatients and outpatients with clinically suspected acute pulmonary embolism were eligible for the study, which was conducted between November 2002 and August 2004 in 12 hospitals in the Netherlands. The institutional review boards of all participating hospitals approved the study protocol. The results and the inclusion and exclusion criteria were published previously. ${ }^{2}$ The study population comprised 3306 patients.

All patients underwent a sequential diagnostic investigation, consisting of clinical probability calculation, a D-dimer test (Tinaquant, Roche Diagnostica, Mannheim, Germany or Vidas D-Dimer Exclusion, Biomerieux) and computed tomography scanning. At admission, the clinical probability of pulmonary embolism was calculated by the treating physician using the Wells score. According to the protocol, a Ddimer test was performed only in patients with a Wells score of $\leq 4$. Pulmonary embolism was ruled out by $(a)$ an unlikely clinical probability (Wells score $\leq 4$ ) combined with a D-dimer test $\leq 500 \mu \mathrm{g} / \mathrm{l}$ or $(b)$ a negative helical computed tomogram in patients with a "likely" clinical probability or an abnormal D-dimer test. Pulmonary embolism was established by a positive helical computed tomogram. The follow-up was performed the same way as in the derivation set studies.

\section{Validation set 2}

For the second validation set, data from a fourth prospective multicentre study were used. ${ }^{3}$ This study investigated in a randomised non-inferiority trial whether the addition of venous ultrasonography to multi-detector computed tomography improved the detection of pulmonary embolism. Consecutive outpatients with clinically suspected acute pulmonary embolism were eligible for the study, which was conducted between January 2005 and August 2006 in six hospitals in France, Belgium, and Switzerland. The institutional review boards of all participating hospitals approved the study protocol. The results and inclusion and exclusion criteria were published previously. ${ }^{8}$ The study population comprised 1812 patients.

All patients underwent a sequential diagnostic investigation, consisting of clinical probability calculation, a D-dimer test (Vidas D-Dimer Exclusion, Biomerieux) and then randomisation to either multi-detector computed tomography alone or compression ultrasonography of the legs followed by computed tomography. At admission, the clinical probability was calculated by the treating physician using the revised Geneva score. ${ }^{15}$ Pulmonary embolism was ruled out by $(a)$ a non-high clinical probability (revised Geneva score $<11$ ) combined with a D-dimer test $<500 \mu \mathrm{g} / \mathrm{l}$ or $(b)$ a negative helical computed tomography result in patients with a high revised Geneva score or an 
Table 1|Baseline characteristics of the derivation and validation cohorts of patients with clinically suspected pulmonary embolism used to produce an age dependent cut-off value for the D-dimer test for pulmonary embolism. Values are numbers (percentages) of patients unless specified otherwise

\begin{tabular}{lccc}
$\begin{array}{l}\text { Characteristic } \\
\text { Age (years): }\end{array}$ & $\begin{array}{c}\text { Derivation set } \\
(\mathrm{n}=1721)\end{array}$ & $\begin{array}{c}\text { Validation set 1 } \\
(\mathrm{n}=3306)\end{array}$ & $\begin{array}{c}\text { Validation set 2 } \\
(\mathrm{n}=1819)\end{array}$ \\
\hline Mean (SD) & $61(19)$ & $53(18)$ & $59(19)$ \\
\hline Median (interquartile range) & $63(45-76)$ & $51(39-68)$ & $61(45-75)$ \\
\hline Female & $1016(59)$ & $1896(57)$ & $922(51)$ \\
\hline History of venous thromboembolism & $308(18)$ & $480(15)$ & $300(17)$ \\
\hline Active malignancy & $164(10)$ & $474(14)$ & $127(7.0)$ \\
\hline Recent surgery & $94(5.5)$ & $46(1.4)$ & $94(5.2)$ \\
\hline Haemoptysis & $80(4.6)$ & $176(5.3)$ & $83(4.6)$ \\
\hline Heart rate $>100$ beats/min & $362(21)$ & $867(26)$ & $369(20)$ \\
\hline Clinical signs of deep vein thrombosis & $246(14)$ & $190(5.7)$ & $153(8.4)$
\end{tabular}

abnormal D-dimer test result. Pulmonary embolism was established by a positive result from helical computed tomography or compression ultrasonography. The follow-up was performed in the same way as the previous studies.

\section{Data analysis}

In the derivation set and validation set 1 the Wells clinical prediction rule for pulmonary embolism was calculated in all patients, and patients were classified according to the dichotomised Wells score as likely or unlikely to have pulmonary embolism. In validation set 2, the revised Geneva score was calculated in all patients and classified as a high or non-high (low or intermediate) score.

To derive a new $\mathrm{D}$-dimer cut-off value, we divided patients aged $>50$ in the derivation set into 10 year age groups. We constructed receiver operating characteristics (ROC) curves of the D-dimer test for each age group to find the best cut-off value (with a sensitivity of $100 \%$ and the highest corresponding specificity). We plotted the D-dimer cut-off level against age group and performed linear regression analysis to obtain the regression coefficient, representing the increase in Ddimer cut-off value per decade. This coefficient was divided by 10 to find the coefficient per year. This coefficient was the multiplication factor for age in the new age-adjusted cut-off value.

We then calculated the proportion of patients with a negative D-dimer test result (as defined by the new cutoff point), the proportion in whom pulmonary embolism could be excluded (based on an "unlikely" Wells score or "non-high" revised Geneva score plus the negative $\mathrm{D}$-dimer test result), and the false negative rates (that is, those patients who had pulmonary embolism in the diagnostic investigation or during followup). The number of patients needed to test by Ddimer to rule out one pulmonary embolism was computed as 1 divided by the proportion of patients with a negative $\mathrm{D}$-dimer test result in each age group. ${ }^{16}$

In the validation sets, D-dimer test results were missing in a large proportion of patients with a "likely" Wells score or high revised Geneva score. Therefore, the age adjusted D-dimer cut-off point was validated only in patients with an "unlikely" or non-high clinical probability score in these two cohorts.

We calculated exact 95\% confidence intervals using CIA software version 1.0 (Gardner et al. Confidence Interval Analysis (CIA), BMJ Books). All other analyses were performed with SPSS version 15.0 (SPSS, Chicago, IL).

\section{RESULTS}

\section{Derivation of the new cut-off value}

Of the 1721 patients included in the derivation set, 416 $(24.2 \%)$ had pulmonary embolism. The D-dimer test was not performed for nine patients from the second study with high clinical probability (all nine had pulmonary embolism diagnosed during initial investigation). Table 1 shows the clinical characteristics of the patients in the derivation cohort.

The figure shows the increase in optimal D-dimer cut-off value by patient age group, obtained from the ROC curves for each group. The cut-off point increased from $512 \mu \mathrm{g} / \mathrm{l}$ in patients $<50$ years old to $934 \mu \mathrm{g} / \mathrm{l}$ in patients $>80$ years old. The regression coefficient was 112 (SE 12.03) $\mu \mathrm{g} / \mathrm{l}$ increase per decade, or $11.2 \mu \mathrm{g} / \mathrm{l}$ increase per year $\left(r^{2}=0.966\right)$. To be conservative and to facilitate clinical usefulness and practicality, we considered a $10 \mu \mathrm{g} / \mathrm{l}$ increase per patient year to be an appropriate new D-dimer coefficient. Starting from the conventional cut-off point of $500 \mu \mathrm{g} / \mathrm{l}$ until the age of 50 , for older patients the patient age should be multiplied by 10 to calculate the age adjusted cut-off value.

\section{Derivation set outcome}

With the conventional cut-off value, the VIDAS Ddimer test was normal $(<500 \mu \mathrm{g} / \mathrm{l})$ in $512 / 1712$ patients (29.9\%), and none had pulmonary embolism during the initial investigation or the three month follow-up $(0 \%, 95 \%$ CI $0 \%$ to $0.7 \%)$. The number of patients needed to test to find one normal D-dimer test result was 3.3. The Wells score could not be computed in 54 of the patients, mainly because information on the likelihood of an alternative diagnosis to that of pulmonary embolism was missing ( $\mathrm{n}=42)$.

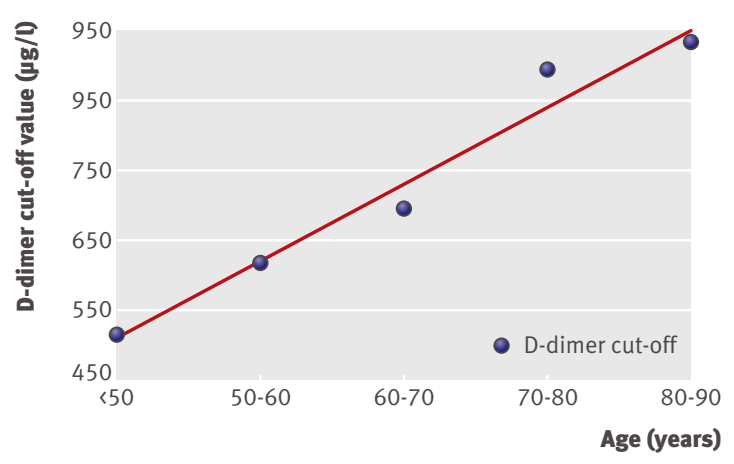

Fig 1 1 Optimal cut-off values for D-dimer test for pulmonary embolism by age in patients with an unlikely clinical probability of pulmonary embolism (sensitivity set at $100 \%$ ) 
Table 2|Proportion of patients in the derivation set with an unlikely clinical probability of pulmonary embolism* in whom pulmonary embolism could be excluded based on a D-dimer test result below the cut-off value: comparison of different cutoff values stratified by age group

\begin{tabular}{|c|c|c|c|c|c|}
\hline & \multirow[b]{2}{*}{ All patients } & \multicolumn{4}{|c|}{ Age range (years) } \\
\hline & & $51-60$ & $61-70$ & $71-80$ & $>80$ \\
\hline No (\%) of patients & 1331 & $189(14)$ & $211(16)$ & $265(20)$ & 198 (15) \\
\hline Median (IQR) age (years) & $61(44-75)$ & $56(54-58)$ & $66(63-68)$ & $76(73-78)$ & $85(82-88)$ \\
\hline \multicolumn{6}{|l|}{ Conventional cut-off value $†$} \\
\hline No $(\%, 95 \% \mathrm{Cl})$ of patients below cut-off value: & $477(36,33$ to 39$)$ & $97(51,44$ to 58$)$ & $63(30,24$ to 36$)$ & $40(15,11$ to 20$)$ & $11(6,3$ to 10$)$ \\
\hline With false negative result & $0(0,0$ to 0.8$)$ & $0(0,0$ to 3.8$)$ & $0(0,0$ to 5.8$)$ & $0(0,0$ to 8.8$)$ & $0(0,0$ to 26$)$ \\
\hline Number needed to test $\ddagger$ & 2.8 & 1.9 & 3.3 & 6.6 & 18 \\
\hline \multicolumn{6}{|l|}{ Age adjusted cut-off value† } \\
\hline No $(\%, 95 \% \mathrm{Cl})$ of patients below cut-off value: & $560(42,39$ to 45$)$ & $102(54,47$ to 61$)$ & $76(36,30$ to 43$)$ & $75(28,23$ to 34$)$ & $41(21,16$ to 27$)$ \\
\hline With false negative result & $1(0.2,0$ to 1.0$)$ & $0(0,0$ to 3.6$)$ & $0(0,0$ to 4.8$)$ & $1(1.3,0.2$ to 7.2$)$ & $0(0,0$ to 8.6$)$ \\
\hline Number needed to test $\ddagger$ & 2.4 & 1.9 & 2.8 & 3.5 & 4.8 \\
\hline \multicolumn{6}{|l|}{$\begin{array}{l}\text { Increase in percentage of patients below } \\
\text { cut-off value: }\end{array}$} \\
\hline Absolute & 6.3 & 2.6 & 6.2 & 13 & 15 \\
\hline Relative & 17 & 5.2 & 21 & 67 & 273 \\
\hline
\end{tabular}

$\mathrm{IQR}=$ interquartile range

*Based on Wells clinical decision rule.

†Conventional cut-off value for D-dimer test $=500 \mu \mathrm{g} /$, age adjusted cut-off value=(age×10) $\mu \mathrm{g} / \mathrm{l}$ (if age >50).

$\ddagger$ Number needed to test to find one normal D-dimer test result

Using the age adjusted cut-off value $($ age $($ years $) \times 10)$ $\mu \mathrm{g} / \mathrm{l})$, we found that $\mathrm{D}$-dimer test results were negative in $615 / 1712$ patients $(46.2 \%$, number needed to test 2.2). This resulted in a $20.1 \%(95 \%$ CI $16.9 \%$ to $23.8 \%)$ relative increase in the number of patients in whom $\mathrm{D}$ dimer levels were considered normal. Of these 615 patients, five had pulmonary embolism during investigation or three month follow-up $(0.8 \%, 0.4 \%$ to $1.9 \%)$.

Table 2 shows the proportion of the 1331 patients with an unlikely clinical probability in whom pulmonary embolism could be excluded based on the conventional and the age adjusted D-dimer cut-off values. There was a $17.4 \%$ (95\% CI $14.3 \%$ to $21.1 \%)$ increase in the number of patients with a negative $\mathrm{D}$-dimer test result when the age adjusted cut-off value was used. The false negative rate was $0(0 \%, 0 \%$ to $0.8 \%)$ for the conventional cut-off value compared with $1(0.2 \%, 0 \%$ to $1.0 \%$ ) for the age adjusted cut-off value. Table 2 also shows the increase in the proportion of patients with an unlikely clinical probability in whom D-dimer levels would be considered normal for specific age groups by using the age adjusted cut-off value: this increase was highest among the oldest patient groups ( $>70$ years), with an absolute increase of $14 \%$ compared with the conventional cut-off point.

\section{External validation}

The concept of using an increasing cut-off value for the D-dimer test according to age was validated in two independent cohorts of patients with suspected pulmonary embolism. The clinical characteristics of the patients in these cohorts were similar to those of the patients in the derivation set (table 1).

Validation set 1

Of the 3306 patients included in validation set 1, 674 $(20.4 \%)$ had pulmonary embolism. In 41 of the 2206 patients with an unlikely clinical probability of pulmonary embolism, D-dimer test results were recorded only qualitatively and were therefore missing for this analysis. In another seven patients age was not documented, leaving 2158 patients. Among these, 983 patients had a negative $\mathrm{D}$-dimer test result with the conventional cut-off value, of whom two $(0.2 \%, 95 \%$ CI $0.1 \%$ to $0.7 \%$ ) had pulmonary embolism during the diagnostic investigation or three month follow-up. With the age adjusted cut-off value, 1093 patients had a negative D-dimer test result, of whom seven $(0.6 \%$, $0.3 \%$ to $1.3 \%$ ) had pulmonary embolism. Table 3 shows the proportion of patients in whom pulmonary embolism could be excluded based on the old and the new cut-off values.

The age adjusted cut-off value resulted in an $11.2 \%$ $(9.3 \%$ to $13.3 \%)$ increase in the number of patients with a negative $\mathrm{D}$-dimer test result. The increase in the proportion of patients in whom pulmonary embolism could be ruled out (that is, unlikely clinical probability with a D-dimer level below the cut-off) from using the age adjusted cut-off value was most prominent among patients in the age groups $>70$ years, with an absolute increase of $16 \%$ (table 3).

The data for validation set 1 came from a study in which two different D-dimer tests were used. ${ }^{2} \mathrm{We}$ therefore performed separate analyses for the two Ddimer tests. There was no difference between the two tests in the false negative rate for the age adjusted Ddimer cut-off value (table 4 ).

\section{Validation set 2}

Of the 1812 patients included in the second validation set, $375(20.7 \%)$ had pulmonary embolism. Among the 1643 patients who had a non-high revised Geneva score, 561 patients $(34 \%$, number needed to test 2.9 ) had a normal D-dimer test result according to the 
Table 3 |Proportion of patients in validation set 1 with an unlikely clinical probability of pulmonary embolism* in whom pulmonary embolism could be excluded based on a D-dimer test result below the cut-off value: comparison of different cutoff values stratified by age group

\begin{tabular}{|c|c|c|c|c|c|}
\hline & All patients & \multicolumn{4}{|c|}{ Age range (years) } \\
\hline No (\%) of patients & 2158 & $358(17)$ & $270(13)$ & $245(18)$ & $166(7.7)$ \\
\hline \multicolumn{6}{|l|}{ Conventional cut-off value $†$} \\
\hline No $(\%, 95 \% \mathrm{Cl})$ of patients below cut-off value: & $983(46,44$ to 48$)$ & $160(45,40$ to 50$)$ & $69(26,21$ to 31$)$ & $40(16,12$ to 22$)$ & $25(15,10$ to 21$)$ \\
\hline Number needed to test & 2.2 & 2.2 & 3.9 & 6.1 & 6.6 \\
\hline \multicolumn{6}{|l|}{ Age adjusted cut-off value $†$} \\
\hline No $(\%, 95 \% \mathrm{Cl})$ of patients below cut-off value: & $1093(51,49$ to 53$)$ & $179(50,45$ to 55$)$ & $96(36,30$ to 41$)$ & $81(33,28$ to 39$)$ & $48(29,23$ to 36$)$ \\
\hline With false negative result & $7(0.6,0.3$ to 1.3$)$ & $1(0.6,0.1$ to 3.1$)$ & $2(2.1,0.6$ to 7.3$)$ & $1(1.2,0.2$ to 6.7$)$ & $1(2.1,0.4$ to 11$)$ \\
\hline Relative & 11 & 12 & 39 & 103 & 92 \\
\hline
\end{tabular}

$\mathrm{IQR}=$ interquartile range

*Based on Wells clinical decision rule.

†Conventional cut-off value for D-dimer test $=500 \mu \mathrm{g} / \mathrm{l}$, age adjusted cut-off value=(age×10) $\mu \mathrm{g} / \mathrm{l}$ (if age $>50$ ).

$\ddagger$ Number needed to test to find one normal D-dimer test result

conventional cut-off value (table 5$)$, and none $(0 \%$, $95 \%$ CI $0 \%$ to $0.7 \%$ ) had pulmonary embolism during the initial investigation or three month follow-up. With the age adjusted cut-off value, $663 / 1643$ patients had a negative D-dimer test result $(40.4 \%$, number needed to test 2.5) (table 5). This resulted in an $18.2 \%(15.0 \%$ to $21.4 \%)$ increase in the number of patients in whom the $\mathrm{D}$-dimer test was negative. The false negative rate with the age adjusted cut-off value was $2(0.3 \%, 0.1 \%$ to $1.1 \%)$. Again use of the age adjusted cut-off value had the greatest effect in the older age groups (table 5).

\section{DISCUSSION}

The study shows that an age adjusted cut-off level for the D-dimer test for exclusion of pulmonary embolism doubles the proportion of older patients ( $>70$ years) in whom pulmonary embolism can be safely excluded in comparison with the conventional cut-off value of $500 \mu \mathrm{g} / \mathrm{l}$. We derived and validated this new cut-off value in three large cohorts of consecutive patients with suspected pulmonary embolism, totalling over 5000 patients. In these three cohorts, the number of

Table $4 \mid$ Proportion of patients in validation set 1 with an unlikely clinical probability of pulmonary embolism* in whom pulmonary embolism could be excluded based on a D-dimer test result below the cut-off value: comparison of the two D-dimer tests used in the set

\begin{tabular}{|c|c|c|c|}
\hline & \multicolumn{2}{|c|}{ D-dimer test } & \multirow{2}{*}{$\begin{array}{l}\text { P value of } \\
\text { difference }\end{array}$} \\
\hline & Tinaquant & Vidas & \\
\hline \multicolumn{4}{|l|}{ Conventional cut-off value for D-dimer test $†$} \\
\hline No (\%) of patients below cut-off value & $576 / 1204(48)$ & $407 / 954(43)$ & 0.016 \\
\hline No $(\%, 95 \% \mathrm{Cl})$ of patients with false negative result & $2 / 576(0.3,0.1$ to 1.3$)$ & $0 / 407(0,0$ to 0.9$)$ & $>0.05$ \\
\hline \multicolumn{4}{|l|}{ Age adjusted cut-off valuefor D-dimer test $†$} \\
\hline No (\%) of patients below cut-off value & $637 / 1204(53)$ & $456 / 954(48)$ & 0.018 \\
\hline No $(\%, 95 \% \mathrm{Cl})$ of patients with false negative result & $4 / 637(0.6,0.2$ to 1.6$)$ & $3 / 456(0.7,0.2$ to 1.9$)$ & $>0.05$ \\
\hline
\end{tabular}

older patients in which pulmonary embolism could be safely ruled out was consistent, between $25 \%$ and $30 \%$.

The clinical usefulness of the new cut-off value increased significantly with age: the proportion of patients in whom pulmonary embolism could be ruled out with the new cut-off value was a third higher in patients older than 50 and almost twice as high in patients older than 70 compared with the conventional cut-off. Increasing the cut-off point to improve clinical utility did not come at the expense of safety: in the derivation and validation sets there was no difference in the false negative rate, and for the total population and for patients aged $>50$ years the $95 \%$ upper confidence levels were well below $3 \%$ with the new cut-off value.

\section{Impact in the clinical setting}

A recent cost effectiveness analysis showed that D-dimer measurement as part of the diagnostic investigation of patients with suspected pulmonary embolism was cost saving until the age of 79 years. ${ }^{17}$ After 80 years, the test's clinical utility was too low to be cost effective, and the costs of strategies with or without Ddimer testing were similar. This analysis was based on the conventional cut-off point of $500 \mu \mathrm{g} / \mathrm{l}$ for ELISA based assays. It can be expected, however, that the test's cost effectiveness in older patients would increase with the new cut-off value, as the number needed to test was lower with the age adjusted cut-off value compared with the conventional cut-off value in patients $>80$ years old (3.5 versus 6.6 ). For a given clinical setting, this means that for every 35 patients aged $>80$ with a low/intermediate or "unlikely" clinical probability of pulmonary embolism, imaging tests can be avoided in five patients when the conventional cut-off is used compared with 10 patients when the age adjusted cut-off value is used. Avoiding imaging tests (that is, ventilation-perfusion (V/Q) scintigraphy or 
Table 5 Proportion of patients in validation set 2 with a non-high clinical probability of pulmonary embolism* in whom pulmonary embolism could be excluded based on a D-dimer test result below the cut-off value: comparison of different cutoff values stratified by age group

\begin{tabular}{|c|c|c|c|c|c|}
\hline & \multirow[b]{2}{*}{ All patients } & \multicolumn{4}{|c|}{ Age range (years) } \\
\hline & & $51-60$ & $61-70$ & $71-80$ & $>80$ \\
\hline No (\%) of patients & 1643 & $252(15)$ & $260(16)$ & $333(20)$ & $253(15)$ \\
\hline Median (IQR) age (years) & $61(45-75)$ & $55(52-57)$ & $65(62-67)$ & $75(72-77)$ & $83(81-86)$ \\
\hline \multicolumn{6}{|l|}{ Conventional cut-off value $†$} \\
\hline No (\%, $95 \% \mathrm{Cl}$ ) of patients below cut-off value: & $561(34,32$ to 37$)$ & $110(44,38$ to 50$)$ & $79(30,25$ to 36$)$ & $43(13,10$ to 17$)$ & $22(9,6$ to 13$)$ \\
\hline With false negative result & $0(0,0$ to 0.7$)$ & $0(0,0$ to 3.4$)$ & $0(0,0$ to 4.6$)$ & $0(0,0$ to 8.2$)$ & $0(0,0$ to 15$)$ \\
\hline Number needed to test $\ddagger$ & 2.9 & 2.3 & 3.3 & 7.7 & 12 \\
\hline \multicolumn{6}{|l|}{ Age adjusted cut-off value $†$} \\
\hline No $(\%, 95 \% \mathrm{Cl})$ of patients below cut-off value: & $663(40,38$ to 43$)$ & $115(46,40$ to 52$)$ & $97(37,32$ to 43$)$ & $89(27,22$ to 32$)$ & $54(21,17$ to 27$)$ \\
\hline With false negative result & $2(0.3,0.1$ to 1.1$)$ & $1(0.9,0.2$ to 4.8$)$ & $0(0,0$ to 3.8$)$ & $1(1.1,0.2$ to 6.1$)$ & $0(0,0$ to 6.6$)$ \\
\hline Number needed to test $\ddagger$ & 2.5 & 2.2 & 2.7 & 3.7 & 4.7 \\
\hline \multicolumn{6}{|l|}{$\begin{array}{l}\text { Increase in percentage of patients below } \\
\text { cut-off value: }\end{array}$} \\
\hline Absolute & 6.2 & 2.0 & 6.9 & 14 & 13 \\
\hline Relative & 18 & 4.5 & 23 & 107 & 145 \\
\hline
\end{tabular}

$\mathrm{IQR}=$ interquartile range

*Based on revised Geneva score $\ll 11$.

†Conventional cut-off value for D-dimer test $=500 \mu \mathrm{g} / \mathrm{l}$, age adjusted cut-off value=(age×10) $\mu \mathrm{g} / \mathrm{l}$ (if age >50).

$\ddagger$ Number needed to test to find one normal D-dimer test result

computed tomography) would be of particular benefit for older patients because of the high frequency of nonconclusive scintigraphy results, the risk from injection with iodine contrast agent for computed tomography scanning, and the length of hospital stay when ordering imaging tests in this patient population.

Some may argue that having to calculate a D-dimer cut-off value is impractical in a clinical setting, and that the new value is really a series of multiple cut-off points. However, the treating physician needs to remember only the coefficient of 10 in order to calculate the new cut-off value, which is an easy multiplication.

\section{Strengths and limitations of study}

The calculation of the age adjusted cut-off value was facilitated by the large size of the study population, which is a major strength of this analysis. However, some aspects of our study warrant comment. Firstly, two different D-dimer assays were used in the

\section{WHAT IS ALREADY KNOWN ON THIS TOPIC}

The combination of D-dimer measurement and clinical probability assessment is valuable for investigation of suspected pulmonary embolism

D-dimer concentrations increase with age, and the specificity of the D-dimer test is decreased in older patients, making the test less useful for excluding pulmonary embolism in such patients

Raising the cut-off value of the D-dimer test for older patients to points between $600 \mu \mathrm{g} / \mathrm{l}$ and $1000 \mu \mathrm{g} / \mathrm{l}$ increases the test's specificity, but at the cost of safety

\section{WHAT THIS STUDY ADDS}

An age adjusted D-dimer cut-off value combined with clinical probability assessment increased the utility of the D-dimer test for excluding pulmonary embolism among older patients without reducing safety

The new cut-off value has sustained external validation. The next step would be to validate the new cut-off prospectively before implementation in daily practice validation cohorts. Although there was no significant difference between the two tests, and the new cut-off value performed equally well with both assays, the study may not have been sufficiently powered to detect a difference between the two. It is unknown how the new cut-off value will perform in other D-dimer assays. Secondly, although D-dimer tests and the (variables for the) clinical decision rule were collected prospectively, this study was a retrospective analysis. After derivation and independent validation in a completely distinct cohort of patients, the next step would be to validate this cut-off value prospectively in a formal outcome study with patient follow-up.

\section{Conclusions}

In conclusion, a cut-off value adjusted to age combined with clinical probability greatly increased the utility of the $\mathrm{D}$-dimer test for the exclusion of pulmonary embolism among older patients without reducing safety. This new cut-off is therefore clinically relevant and has sustained external validation. The next step would be to validate this new $\mathrm{D}$-dimer cut-off value prospectively before implementation in daily practice.

Contributors: RAD, GIG, PWK, and PMR conceived and designed the study and performed analysis and interpretation of data. MS, MR, AP, and MJHAK collected and interpreted data. RAD drafted the manuscript. All authors were involved in revision and final approval of the manuscript. All authors had full access to the data in the study.

Funding: None

Competing interests: All authors have completed the unified competing interest form at www.icmje.org/coi_disclosure.pdf (available on request from the corresponding author) and declare (1) no financial support for the submitted work from anyone other than their employer; (2) no financial relationships with commercial entities that might have an interest in the submitted work; (3) no spouses, partners, or children with relationships with commercial entities that might have an interest in the submitted work; and (4) no non-financial interests that may be relevant to the submitted work.

Ethical approval: Not required.

Data sharing: No additional data available. 
1 Perrier A, Roy PM, Sanchez O, Le Gal G, Meyer G, Gourdier AL, et al. Multidetector-row computed tomography in suspected pulmonary embolism. N Engl J Med 2005;352:1760-8.

2 Van Belle A, Buller HR, Huisman MV, Huisman PM, Kaasjager K, Kamphuisen PW, et al for the Christopher Study Investigators.

Effectiveness of managing suspected pulmonary embolism using an algorithm combining clinical probability, D-dimer testing, and computed tomography. JAMA 2006;295:172-9.

3 Righini M, Le Gal G, Aujesky D, Roy PM, Sanchez O, Verschuren F, et al. Diagnosis of pulmonary embolism by multidetector CT alone or combined with venous ultrasonography of the leg: a randomised non-inferiority trial. Lancet 2008;371:1343-52.

4 Hager K, Platt D. Fibrin degeneration product concentrations (Ddimers) in the course of ageing. Gerontology 1995;41:159-65.

5 Perrier A, Desmarais S, Goehring C, de Moerloose P, Morabia A Unger PF, et al. D-dimer testing for suspected pulmonary embolism in outpatients. Am J Respir Crit Care Med 1997;156:492-6.

6 Tardy B, Tardy-Poncet B, Viallon A, Lafond P, Page Y, Venet C, et al. Evaluation of D-dimer ELISA test in elderly patients with suspected pulmonary embolism. Thromb Haemost 1998;79:38-41.

7 Masotti L, Ceccarelli E, Cappelli R, Forconi S. Plasma D-dimer levels in elderly patients with suspected pulmonary embolism. Thromb Res 2000;98:577-9.

8 Righini M, Goehring C, Bounameaux H, Perrier A. Effects of age on the performance of common diagnostic tests for pulmonary embolism. Am J Med 2000;109:357-61.

9 Sohne M, Kruip MJ, Nijkeuter M, Tick L, Kwakkel H, Halkes SJ, et al. Accuracy of clinical decision rule, D-dimer and spiral computed tomography in patients with malignancy, previous venous thromboembolism, COPD or heart failure and in older patients with suspected pulmonary embolism. J Thromb Haemost 2006;4:1042-6.
10 Wells PS, Anderson DR, Rodger M, Ginsberg JS, Kearon C, Gent M, et al. Derivation of a simple clinical model to categorize patients probability of pulmonary embolism: increasing the models utility with the SimpliRED D-dimer. Thromb Haemost 2000;83:416-20.

11 Righini M, de Moerloose P, Reber G, Perrier A, Bounameaux H. Should the D-dimer cut-off value be increased in elderly patients suspected of pulmonary embolism? Thromb Haemost 2001;85:744.

12 Perrier A, Roy PM, Aujesky D, Chagnon I, Howarth N, Gourdier AL, et al. Diagnosing pulmonary embolism in outpatients with clinical assessment, D-dimer measurement, venous ultrasound, and helical computed tomography: a multicenter management study. Am J Med 2004;116:291-9.

13 Wicki J, Perneger TV, Junod AF, Bounameaux H, Perrier A. Assessing clinical probability of pulmonary embolism in the emergency ward: a simple score. Arch Intern Med 2001;161:92-7.

14 Chagnon I, Bounameaux H, Aujesky D, Roy PM, Gourdier AL, Cornuz I, et al. Comparison of two clinical prediction rules and implicit assessment among patients with suspected pulmonary embolism. Am J Med 2002;113:269-75.

15 Le Gal G, Righini M, Roy PM, Sanchez O, Aujesky D, Bounameaux H, et al. Prediction of pulmonary embolism in the emergency department: the revised Geneva score. Ann Intern Med 2006;144:165-71.

16 Righini M, Aujesky D, Roy PM, Cornuz J, de Moerloose P, Bounameaux $\mathrm{H}$, et al. Clinical usefulness of D-dimer depending on clinical probability and cutoff value in outpatients with suspected pulmonary embolism. Arch Intern Med 2004;164:2483-7.

17 Righini M, Nendaz M, Le Gal G, Bounameaux H, Perrier A. Influence of age on the cost-effectiveness of diagnostic strategies for suspected pulmonary embolism. J Thromb Haemost 2007;5:1869-77.

Accepted: 6 January 2010 